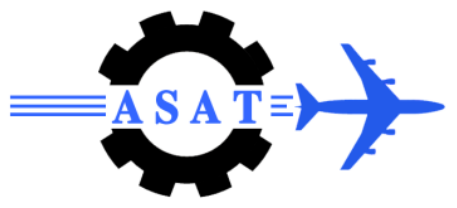

\title{
Peak-to-Average Power Ratio Reduction of Uplink Broadband Transmission Systems using Different Reduction Algorithms
}

\author{
H.E.A. Hassan ${ }^{*}$, F.A.K. Al-fuhaidy ${ }^{\dagger}$, Kh. El-barbary*
}

\begin{abstract}
Partial transmit sequence (PTS) and selective mapping (SLM), are two algorithms used for peak-to-average power ratio (PAPR) reduction in multicarrier transmissions. In this paper, these techniques are applied for single carrier transmission system enhancement. The advantage of these algorithms, that they have no effect on the bit error (BER) of the system. But it will increase the complexity of the system due to the need for additional inverse discrete fourier transform IDFT calculations, search complexity for optimum phase factor. These algorithms are implemented on a single carrier frequency division multiple access (SCFDMA) system. SC-FDMA is used in Long Term Evaluation (LTE) of $3^{\text {rd }}$ Generation Partnership Project (3GPP) as a strong candidate for uplink multiple access scheme. Simulations show that the proposed system with these two algorithms introduces a significant PAPR reduction compared to conventional system. There is a tradeoff between the PAPR reduction and the transmitter complexity. Due to the need for additional IDFT blocks, the complexity increased and due to side information, data rate has small decrease. Different simulation parameters are used for these algorithms which provide a good indication for selection of optimum technique with optimum parameters.
\end{abstract}

Keywords: PTS, SLM, PAPR, IDFT, SC-FDMA, LTE, 3GPP

\section{Introduction}

Demands for media-rich wireless data services have brought much attention to high speed broadband mobile wireless techniques in recent years. Orthogonal frequency division multiplexing (OFDM), which is a multicarrier communication technique, has become widely accepted. That is due to its robustness against frequency selective fading channels that are common in broadband mobile wireless communications [1]. Orthogonal frequency division multiple access (OFDMA), is a multiple access scheme which is an extension of OFDM to accommodate multiple simultaneous users access. Since, SC-FDMA has lower PAPR compared to OFDMA system, SC-FDMA is adopted as a possible air interface, especially in uplink broadband communications [1-2]. SC-FDMA system is the multi-user version of the SC-FDE system. The main advantages of the SC-FDMA system are that the envelope fluctuations are less pronounced and the power efficiency is higher than that of the OFDMA [2]. The high PAPR requires system components with a large linear range capable of accommodating the signal. Otherwise, the nonlinear distortion occurs, which results in a loss of subcarrier orthogonality, and degrades the system performance. Many PAPR reduction techniques for multi-carrier systems were surveyed in [3] which may be used by single-carrier

\footnotetext{
*Egyptian Armed Forces, Egypt, hossameldin_aboubakr@hotmail.com

†Yemen Armed Forces, farouqakh@gmail.com

* Modern Academy, Staff member, khbar2000@yahoo.com.
} 
systems. The clipping algorithm causes signal distortion and performance degradation [4-5], while other algorithms such as PTS and SLM may be not affects the system performance [67]. But, they have an overhead and significantly increased complexity. When the PTS or SLM algorithms are applied the system performance not affected, while complexity of the system increased.

The reminder of this paper is organized as follows: Section 2 provides an overview of SCFDMA. Section 3 explains two different algorithms used for PAPR. Section 4 presents the proposed models and PAPR problem explanation. Section 5 presents the CCDF of PAPR of the proposed system compared to conventional system by simulation and discusses these results. Finally, last section gives the conclusions.

\section{Overview of SC-FDMA System Model}

Single carrier FDMA can be regarded as discrete fourier transform (DFT)-spread OFDMA because, time domain data symbols are transformed to frequency domain by DFT before going through OFDMA modulation [2]. Figure 1 shows the block diagram of a SC-FDMA system.

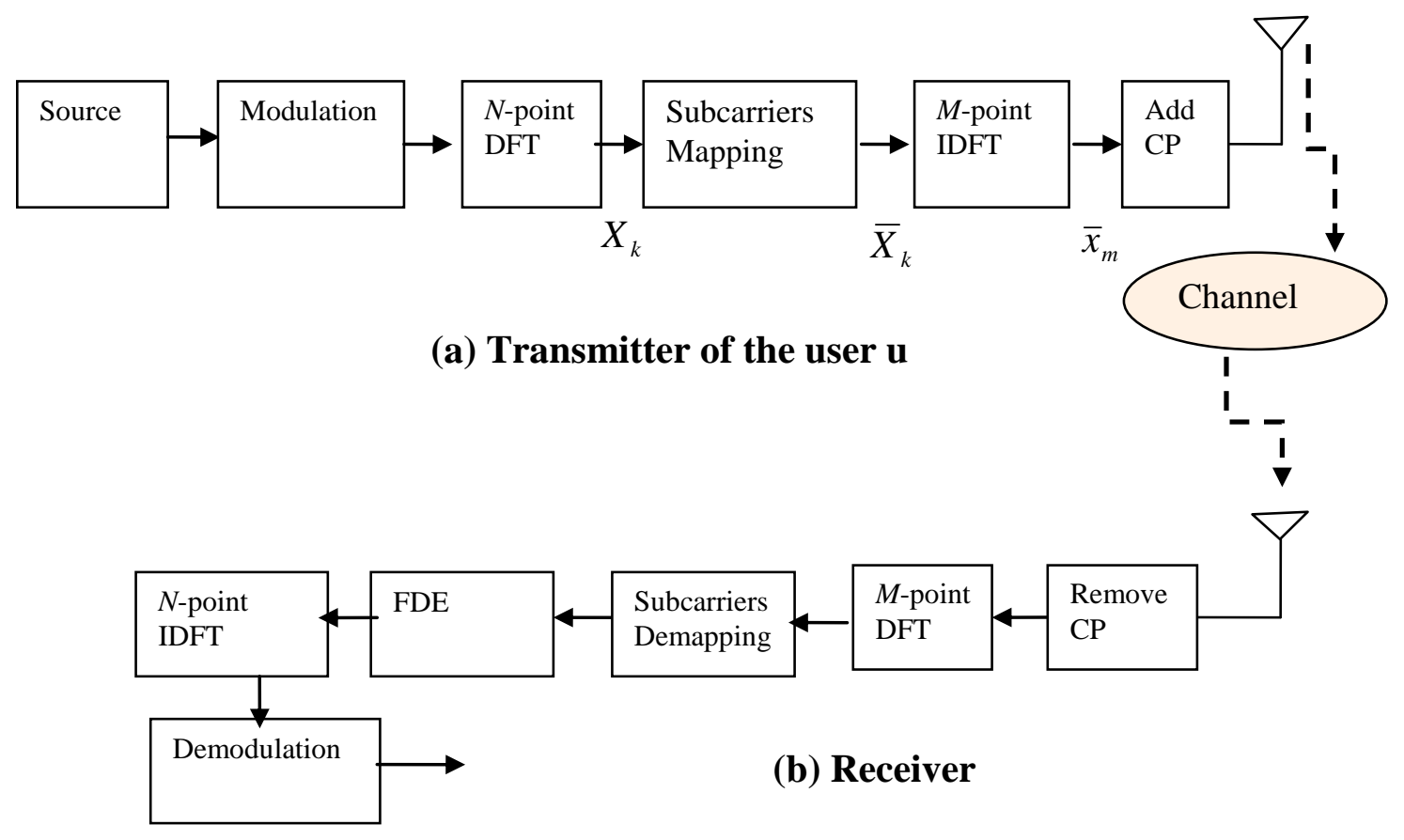

\section{Figure 1 Block diagram of SC-FDMA system over a frequency selective channel.}

At the transmitter, binary input data passes through one of many possible modulation techniques such as QAM, or M-QAM, introducing symbols. A DFT operation performed on each block of $N$ symbols. Then, subcarriers are mapped in the frequency domain. There are three types of mapping, localized, interleaving, or Distributed mapping [8]. The IDFT is performed on each block of $M$ subcarriers. After that, a cyclic prefix CP of $N c$ symbols is added to the resulting signal. The length of the $\mathrm{CP}$ must be greater than the maximum delay spread of the channel to accommodate the inter-block interference (IBI) [2]. At the receiver, the CP is removed from the received signal. An M-point DFT is performed on the received signal, and getting the frequency domain signal. Then, Frequency Domain Equalization (FDE) and subcarrier demapping are performed. An N-point IDFT is performed on the resulting signal. Finally, demodulation is performed. 


\section{The PTS and the SLM Algorithms}

Figure 2 shows the block diagram for PTS algorithm [9]. In PTS, an input data block of $M$ symbols is partitioned into disjoint sub-blocks of same length such as the summation of subblocks is equal to the partitioned block. Each sub-block is passed through IDFT block. Then, multiply each sub-block by a phase factor. The phase factors are selected such that the PAPR of the combined signal is minimized. In general, the selection of the phase factors is limited to a set with a finite number of elements to reduce the search complexity. The set of allowed phase factors is written as $P=\left\{e^{2 \pi l / W} \mid l=0,1, \ldots, W-1\right\}$, where $W$ is the number of allowed phase factors [3]. The amount of PAPR reduction depends on the number of sub-blocks $V$ and the number of allowed phase factors $W$. Another factor that may affect the PAPR reduction performance in PTS is the sub-block partitioning, which is the method of division of block into sub-blocks. There are three kinds of sub-block partitioning schemes: adjacent, interleaved and pseudorandom partitioning. PTS needs $M$ IDFT operations for each data block, and the number of required side information bits is $\left[\log _{2} W^{V-1}\right]$, where $[y]$ denotes the smallest integer that does not exceed $y$.

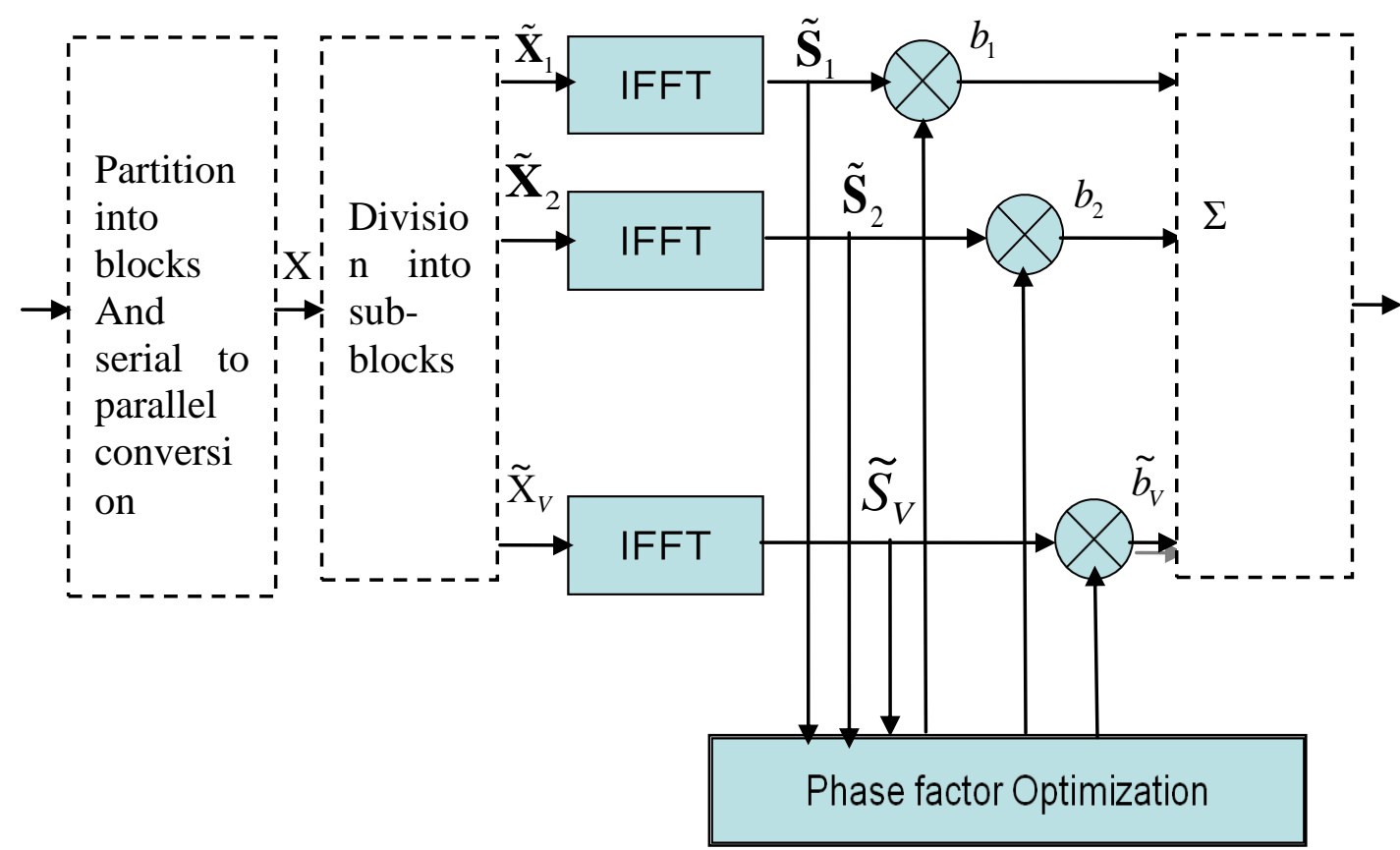

Figure 2 A block diagram of the PTS algorithm

In SLM, the transmitter generates a set of sufficiently different candidate data blocks, all representing the same information as the original data block, and selects the most favorable for transmission [10,11]. A block diagram for SLM is shown in figure 3 which is redrawn from [3]. Each data block is multiplied by $U$ different phase sequences, each of length $M$, $\mathrm{B}^{(\mathrm{u})}=\left[\mathrm{b}_{u, 0}, \mathrm{~b}_{u, 1}, \ldots, \mathrm{b}_{u, M-1}\right]^{T}, u=1,2, \ldots, U$, resulting in $U$ modified data blocks. Then each modified data block passes through IDFT block. Finally, the one with smallest PAPR is selected for transmission. Information about the selected phase sequence should be transmitted to the receiver as side information. At the receiver, reverse operation is performed to recover the original data block. For implementation, the SLM technique needs $U$ IDFT operations, and the number of required side information bits is $\left[\log _{2} U\right]$ for each data block. The amount of PAPR reduction of SLM is depending on the number of phase sequences $U$, and the design of the phase sequences. 


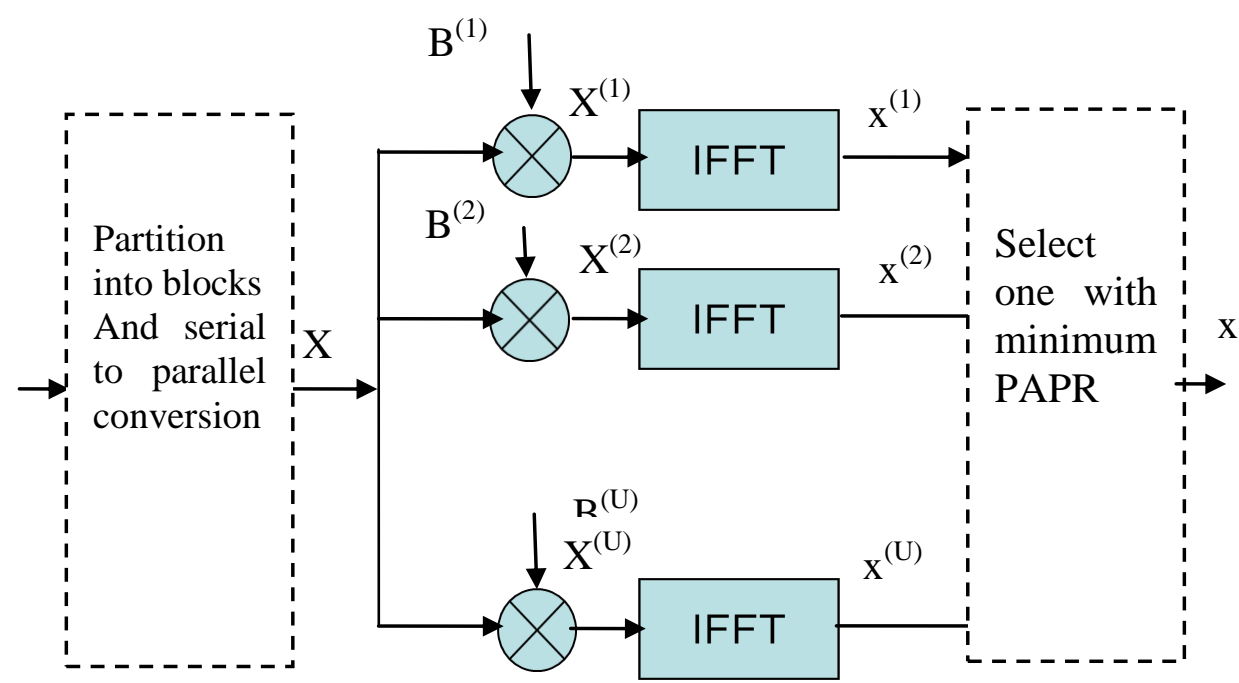

Figure 3 A block diagram of the SLM algorithm

\section{The Proposed SC-FDMA with PTS and SLM Algorithms and PAPR Problem}

There are many PAPR reduction techniques used in multi-carrier transmission systems [3]. In this work, we use two of these techniques, PTS and SLM, for single carrier transmission systems, SC-FDMA. Figures 4.a, 4.b show the block diagram of the transmitter of the proposed SC-FDMA system with PTS and SLM respectively.

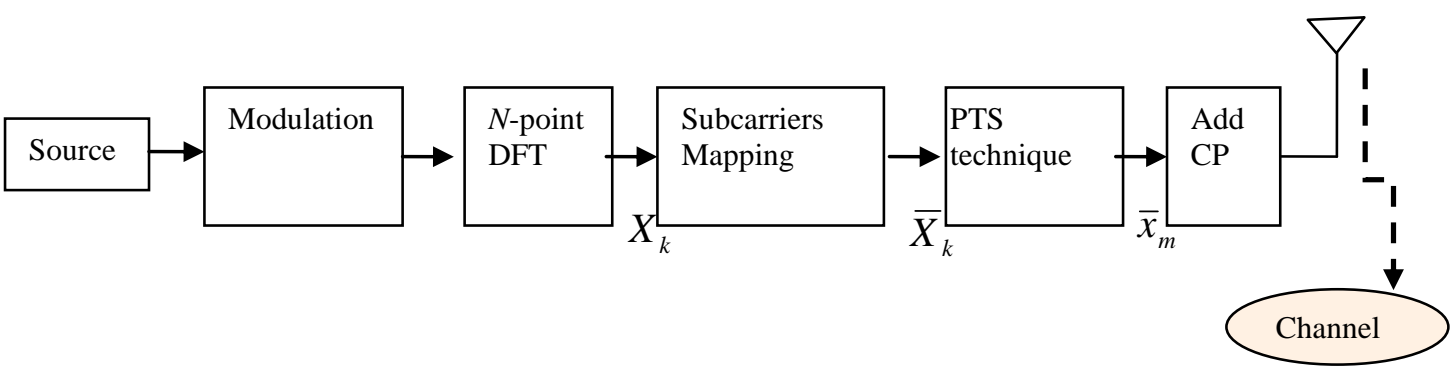

(4.a) SC-FDMA Transmitter with SLM algorithm

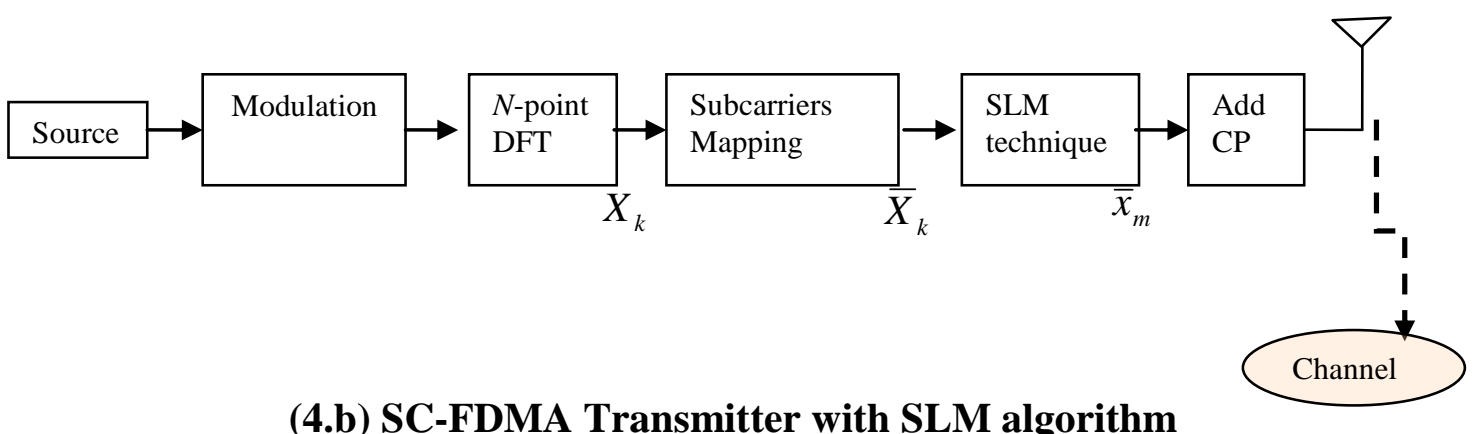

Figure 4 Block diagram of SC-FDMA system over a frequency selective channel. 
Implementation of these techniques with conventional SC-FDMA system as follow:

- The block of symbols resulting from modulating the baseband signal is converted to frequency domain using DFT operation.

- Then, mapping of frequency domain symbols to orthogonal subcarriers is done.

- After that, the PAPR reduction technique, PTS or SLM, is applied which result in a minimized PAPR block of time domain symbols.

- The difference between the works of these techniques is shown below.

The main difference between the two algorithms is that in PTS, each sub-block is multiplied by phase factors in the time domain, and the transmitted block is the sum of these multiplied blocks. In SLM, there are multiple copies of the original block multiplied by different phase factors in frequency domain. Then transmit the block of the minimum PAPR. Since, phase factors are a combination of 1's and -1 's, the original data block can be included using a phase factor of all 1's. The PAPR of the transmitted signal is given by (1) is defined as:

$$
P A P R=\frac{P_{p e a k}}{P_{a v}}=\frac{\left.\left.\max _{0 \leq m \leq M-1}\left(\mid \bar{x}_{m}\right)\right|^{2}\right)}{1 / M \sum_{m=0}^{M-1}\left|\bar{x}_{m}\right|^{2}}
$$

The PAPR in SC-FDMA is required to be as small as possible. The lower PAPR greatly benefits the mobile terminal in terms of transmit power efficiency [2]. The high PAPR causes the peaks to enter the saturation region of the power amplifier (PA). Non-linear amplification by the PA causes in-band distortion which increases the BER at the receiver. The out-off-band distortion causes spectral regrowth. To overcome this problem by using of PA that is highly linear. However, it is very expensive. Most researches use complementary CDF (CCDF) instead of CDF [1-2]. The CCDF of the PAPR denotes the probability that the PAPR of a data block exceeds a given threshold PAPR $>\mathrm{PAPR}_{\mathrm{th}}$.

\section{Computer Simulations}

Computer simulations of the proposed SC-FDMA combined PTS and SLM algorithms are done using Matlab. Localized mapping which is used by LTE in $3^{\text {rd }}$ generation partnership project (3GPP) [12] is implemented. The conventional SC-FDMA scheme is also simulated for comparison purpose. Simulation parameters are listed in Table 1.

Table 1. Simulation Parameters

\begin{tabular}{l|l|l}
\hline \hline & \multicolumn{1}{|c|}{ Description } & \multicolumn{1}{c}{ Parameters } \\
\hline \hline \multirow{4}{*}{ Transmitter } & System bandwidth & $5 \mathrm{MHz}$ \\
\cline { 2 - 3 } & Modulation & $16-\mathrm{QAM}$ \\
\cline { 2 - 3 } & Transmitter IDFT size & $\mathrm{M}=512 \mathrm{symbols}$ \\
\cline { 2 - 3 } & Subcarrier spacing & $9.765625 \mathrm{kHz}$ \\
\cline { 2 - 3 } & SC-FDMA input block size & 128 symbols \\
\cline { 2 - 3 } & Subcarrier mapping & Localized \\
\hline \multirow{4}{*}{ PTS } & Mapping & Adjacent and Interleaved \\
\cline { 2 - 3 } & Number of sub-blocks (V) & 4 and 8 \\
\cline { 2 - 3 } & Number of allowed phase factors (W) & 16,32, and 64 \\
\hline SLM & Number of phase sequences (U) & $4,8,16$, and 32 \\
\hline
\end{tabular}




\section{Peak-to-Average Power Ratio Reduction for SC-FDMA and OFDMA with PTS}

In this subsection, SC-FDMA and OFDMA with and without PTS are simulated. Discussion of the effect of PTS on both systems is shown, in term of CCDF and PAPR. Figure 5 shows the CCDF for PAPR for different partitioning scheme, adjacent and interleaved types. Each block is partitioned into four sub-blocks and QPSK modulation is implemented on SCFDMA. The proposed schemes compared to conventional SC-FDMA.

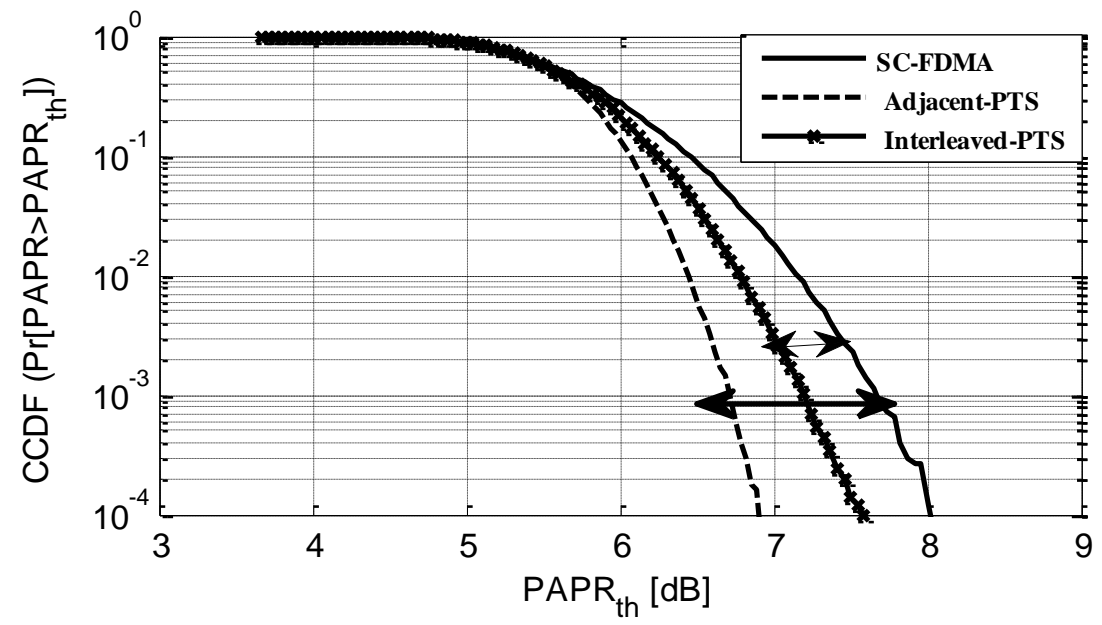

Figure 5 CCDF for PAPR of SC-FDMA with and without PTS, V=4 and QPSK modulation

As shown in above figure, the adjacent partitioning provides approximately twice PAPR reduction compared to interleave partitioning with respect to conventional SC-FDMA. Figure 6 shows simulations for OFDMA system, which has a larger signal fluctuations compared to SC-FDMA.

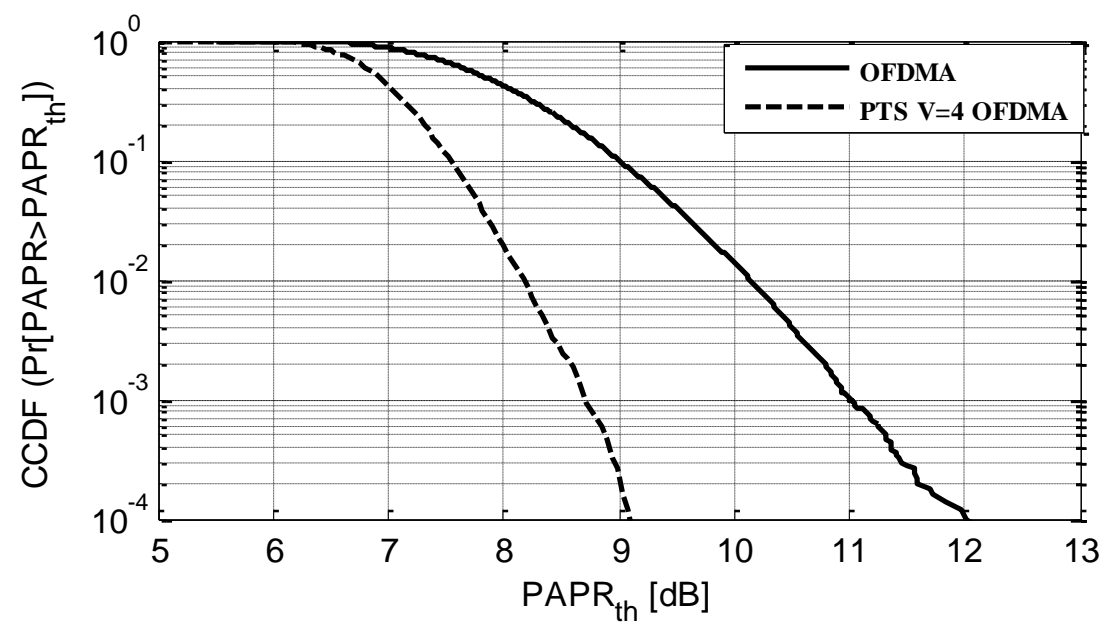

Figure 6 CCDF for PAPR of OFDMA with and without PTS, $V=4$ and QPSK modulation

Figure 6 shows that PTS work well with OFDMA compared to PTS with SC-FDMA. Intuitively, that is what expected. That is due to larger signal fluctuations of OFDMA signals compared to SC-FDMA signal. By comparing Figure 5 for SC-FDMA with PTS to Figure 6 for OFDMA, it is shown that PTS work well with OFDMA due to larger signal fluctuations in 
OFDMA. However, SC-FDMA with PTS has a PAPR smaller than the PAPR of OFDMA with PTS.

Figures 7 and 8 have the same simulation parameters as in figures 5 and 6 respectively, except number of sub-blocks, $V=8$.

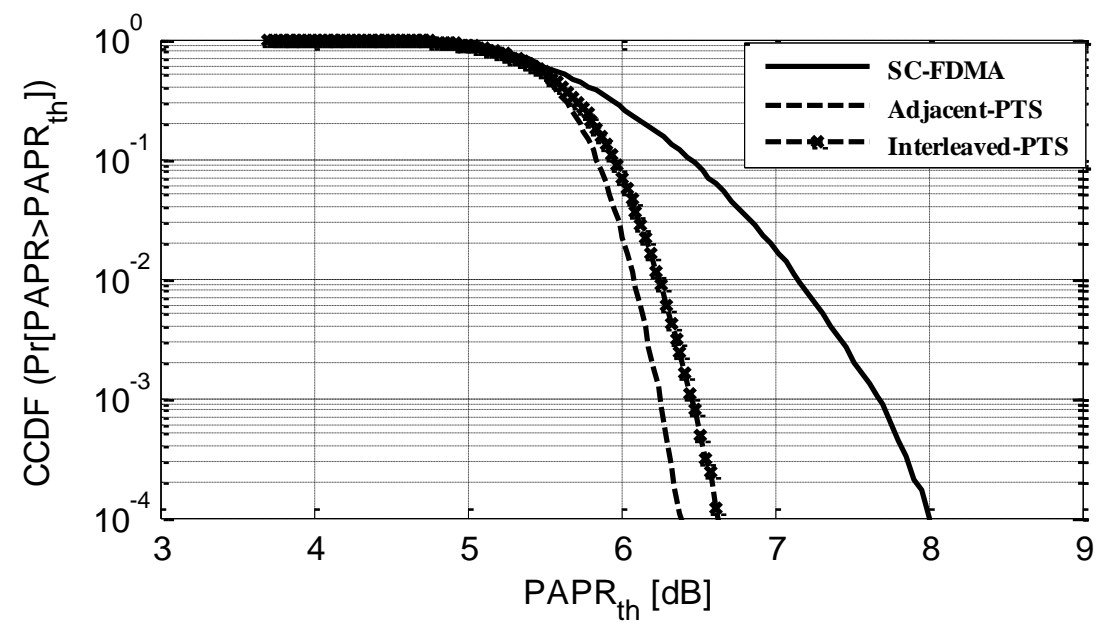

Figure 7 CCDF for PAPR of SC-FDMA with and without PTS, V=8 and QPSK modulation

As shown in previous figure, as the number of sub-blocks increased, the PAPR reduction increased. This is one of the parameters that affect the amount of PAPR reduction. Notice that adjacent partitioning has the best PAPR reduction compared to interleave partitioning.

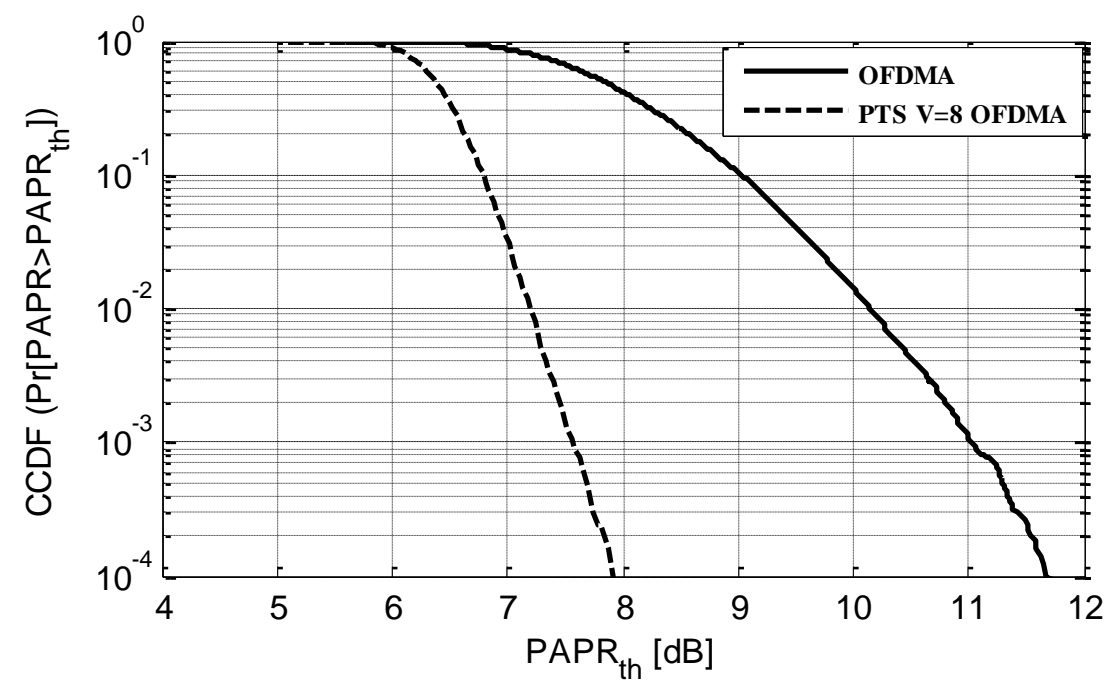

Figure 8 CCDF for PAPR of OFDMA with and without PTS, V=8 and QPSK modulation

It is also shown from previous figure that, PTS work well with OFDMA. But it has a larger PAPR compared to SC-FDMA with PTS algorithm.

Figure 9 studies the effect of number of sub-blocks, used in partitioning of the original block in PTS algorithm. Adjacent partitioning is used and QPSK modulation. 


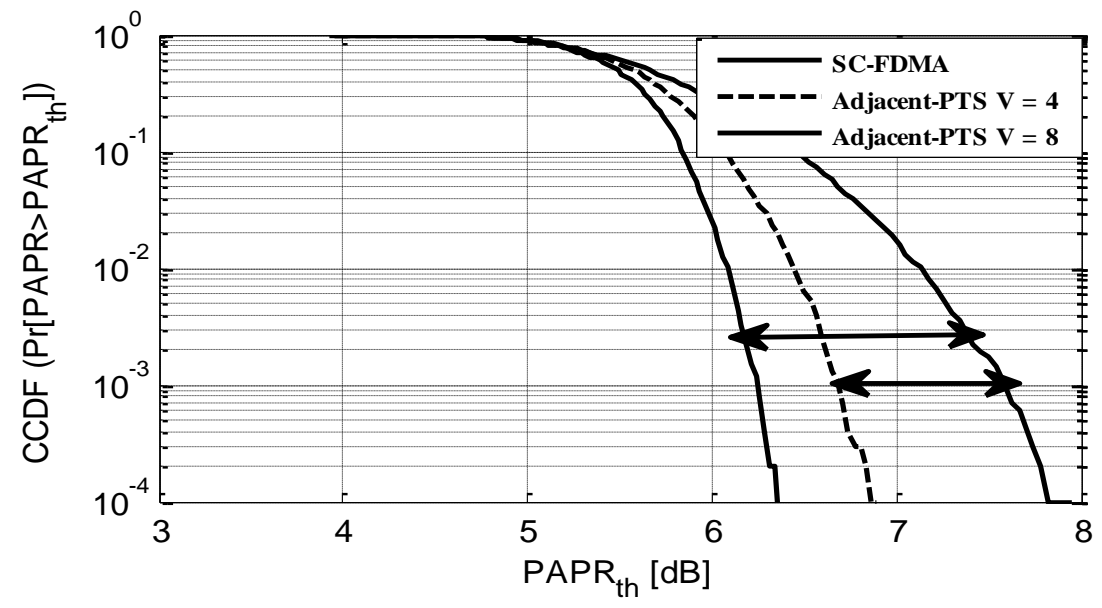

Figure 9 CCDF for PAPR of SC-FDMA with and without PTS, $V=4$ and $V=8$ and QPSK modulation

As shown in Figure 9, as the number of sub-blocks increases, the PAPR reduction increases. However, this increases the cost and complexity by increasing the number of IDFT operations.

\section{Peak-to-Average Power Ratio Reduction for SC-FDMA with SLM}

In this subsection, the implementation and simulation of SLM technique on SC-FDMA is performed using computer simulations. QPSK modulation and different number of phase sequences for SLM with SC-FDMA is shown in Figure 10.

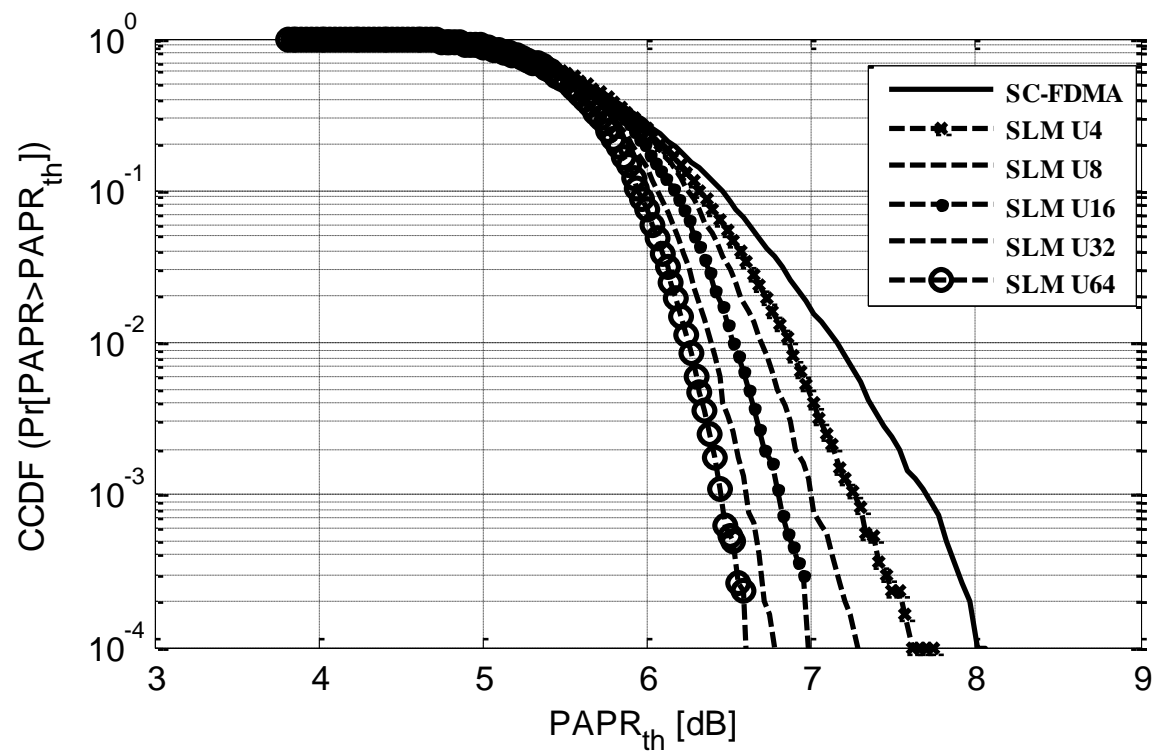

Figure 10 CCDF for PAPR of SC-FDMA with and without SLM, different number of phase sequences $U=4,8,16,32$ and 64 .

It is shown from previous figure, as the number of phase sequences, $U$, increases, the PAPR reduction also increases. However, as the number of phase sequences increases, complexity of the system increases. 
3. PAPR Reduction for SC-FDMA with PTS Compared to SC-FDMA with SLM In this subsection, SLM is compared to PTS via simulations. The effect of each algorithm on the PAPR reduction is shown. This provides the reader which is the best at the same complexity. Figure 11 and Figure 12 compare the two algorithms for number of IDFT blocks set to 4 and 8 respectively. It is worth noting that QPSK modulation is used in these simulations.

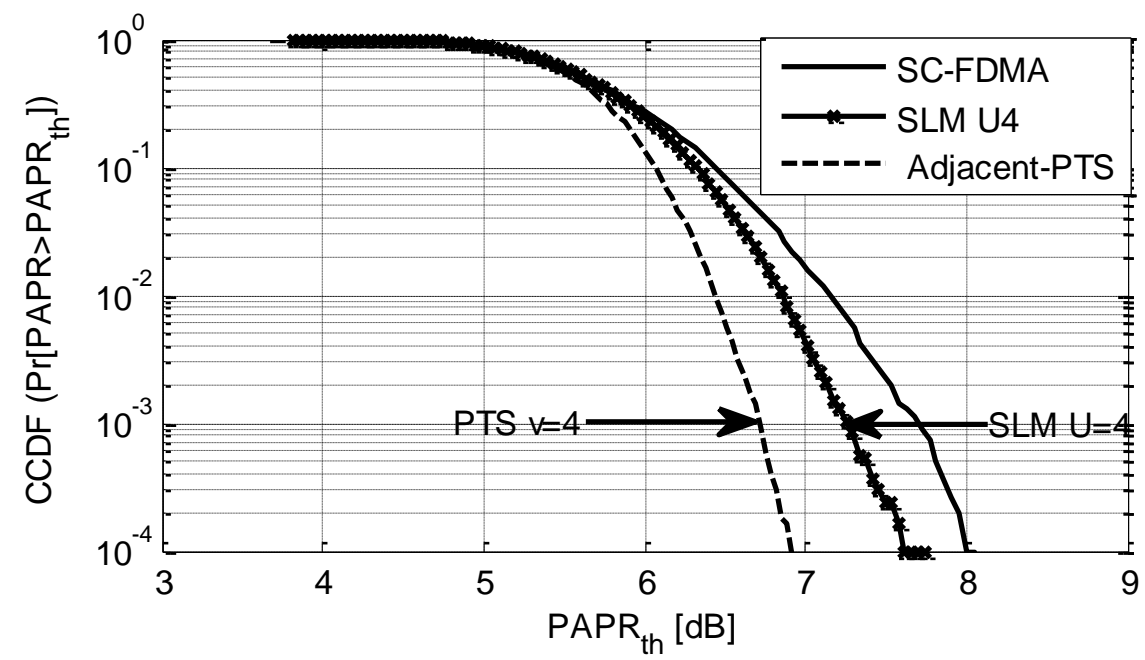

Figure 11 CCDF for PAPR of SC-FDMA with PTS compared to SC-FDMA with SLM, $U=V=4$ and 16QAM modulation

As shown in Figure 11, the PTS algorithm provides approximately twice the gain factor of PAPR reduction compared to SLM algorithm. Number of IDFT blocks of the two algorithms is equal but, PTS algorithm takes some additional cost for the search of the optimum phase factors.

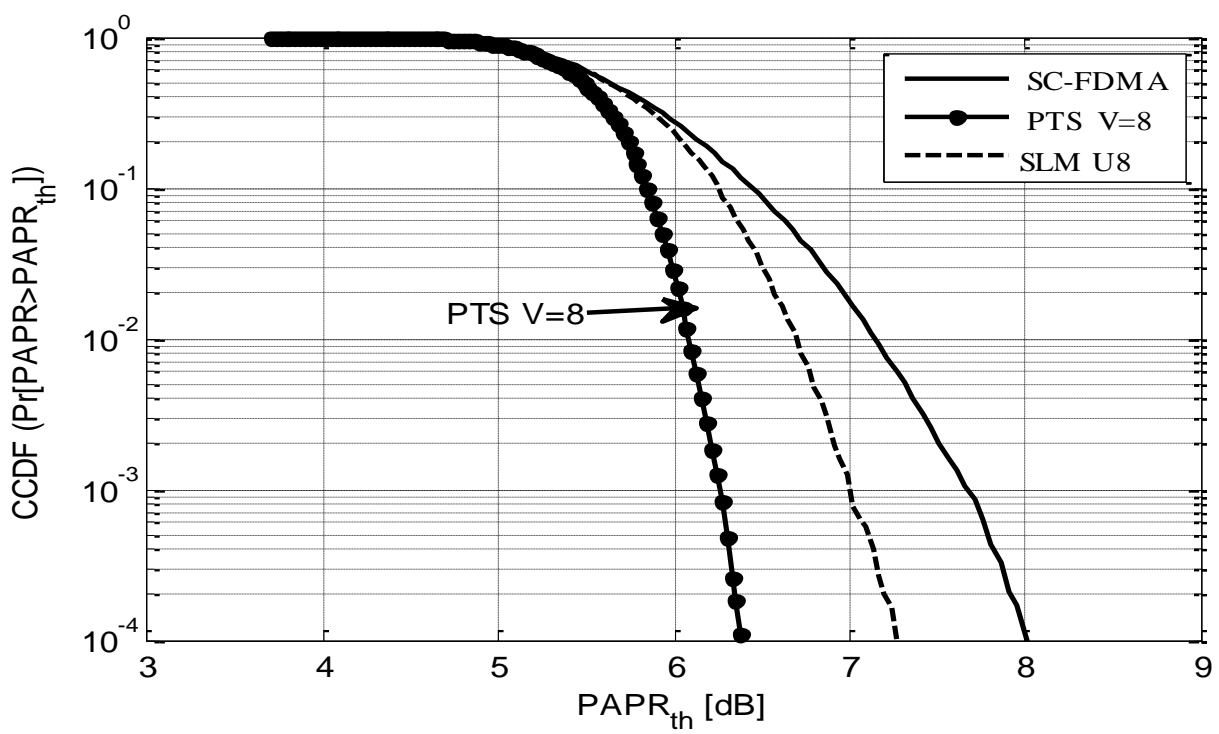

Figure 12 CCDF for PAPR of SC-FDMA with PTS compared to SC-FDMA with SLM, $U=V=8$ and 16QAM modulation

In figure 12, PTS provides a larger PAPR reduction compared to SLM for the same number of IDFT blocks. However, the difference is that the PTS do additional cost in searching of the optimum phase factor. For example in Figure 12 PTS algorithm searches for 64 different phase factors. However, 8 factors are available for SLM. 


\section{Conclusions}

SC-FDMA has a lower PAPR than OFDMA. However, more reduction in PAPR is obtained in this paper by applying different algorithms. This increases the battery life time and improves the power efficiency. In this paper, two different multi-carrier PAPR reduction algorithms are applied for single carrier PAPR reduction. These two algorithms are PTS and SLM. They provide a good PAPR reduction of SC-FDMA system. However, these algorithms increase the complexity and the cost of the transmitter due to the use of additional IDFT blocks. Different simulation parameters are studied in simulation to provide a good indication of the optimum parameters for these algorithms.

\section{References}

[1] H. G. Myung, J. Lim and D. J. Goodman, "Single Carrier FDMA for Uplink Wireless transmission," Proceeding of the IEEE Vehicular Technology Magazine., vol. 1, no. 3, Sep. 2006.

[2] H. G. Myung, J. Lim and D. J. Goodman, "Peak-to-Average power Ratio of Single Carrier FDMA Signals with Pulse Shaping," Proceeding of the IEEE PIMRC, pp. 1-5, Sep. 2006.

[3] S. H. Han and J. H. Lee, "An Overview of Peak-to-Average Power Ratio Reduction Techniques for Multicarrier Transmission," IEEE Wireless Communications, vol. 12, no. 2, 2005, pp. 56-65.

[4] ZID Souad and B. Ridha, "Low-Complexity PAPR Reduction Schemes Using SLM and PTS Approaches for Interleaved OFDMA," IEEE, 2009.

[5] Seng-Hung Wang, Jia-Cheng Xie and Shih-Peng Li, "A Low-Complexity SLM PAPR Reduction Scheme for Interleaved OFDMA Uplink," IEEE GLOBECOM, 2009.

[6] J. Armstrong, "Peak-to-Average Power Reduction for OFDM by Repeated Clipping and Frequency Domain Filtering," Electron. Lett., vol. 38, Feb. 2002, pp. 246-247.

[7] Yue Xiao, Yongrui Peng and Shaoqian Li "PAPR Reduction for Interleaved OFDMA with Low Complexity," ICICS, 2007.

[8] H. G. Myung, J. Lim and D. J. Goodman, "Peak-to-Average power Ratio of Single Carrier FDMA Signals with Pulse Shaping," Proceeding of the IEEE PIMRC, 2006.

[9] S. H. Müller and J. B. Huber, "OFDM with Reduced Peak-to-Average Power Ratio by Optimum Combination of Partial Transmit Sequences," Elect. Lett., vol. 33, no. 5, Feb. 1997, pp. 368-69.

[10] S. H. Müller and J. B. Huber, "A Comparison of Peak Power Reduction Schemes for OFDM," Proc. IEEE GLOBECOM '97, Phoenix, AZ, Nov. 1997, pp. 1-5.

[11] R. W. Bäuml, R. F. H. Fisher, and J. B. Huber, "Reducing the Peak-to-Average Power Ratio of Multicarrier Modulation by Selected Mapping," Elect. Lett., vol. 32, no. 22, Oct. 1996, pp. 2056-57.

[12] H. G. Myung and D. J. Goodman, "Single Carrier FDMA A new Air Interface for Long Term Evaluation," Wiley Series on Wireless Communications and Mobile Computing, 2008. 